\title{
Vi rispondiamo... per il giusto tramite
}

\author{
Sara Giovine
}

PUBBLICATO: 07 FEBBRAIO 2020

\section{Quesito:}

Molti lettori ci scrivono per avere delucidazioni sul corretto impiego della forma tramite: è possibile utilizzarla con funzione di preposizione o, trattandosi di un sostantivo, va inclusa all'interno della più ampia locuzione preposizionale per il tramite di? Ci viene inoltre chiesto se sia preferibile ricorrere alle preposizioni mediante e attraverso e quali siano le eventuali differenze di significato. Infine, qualcuno chiede se esiste la forma plurale tramiti.

\section{Vi rispondiamo... per il giusto tramite}

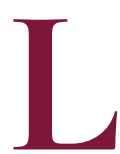

a forma tramite, nel suo valore primario e originario, è in effetti, come sottolineano molti dei nostri lettori, un sostantivo: si tratta infatti di una voce dotta, derivante dal sostantivo latino trames, - itis, letteralmente 'sentiero traverso, passaggio, scorciatoia' (a sua volta dal verbo trameare, composto di meare 'passare' e del prefisso trans 'attraverso'), che, secondo quanto indicato dai principali dizionari etimologici (tra cui il DELI e l'Etimologico), risulta attestata in italiano a partire dal XIV secolo appunto come sostantivo, nel significato di 'passaggio, sentiero, via di comunicazione'.

Nella sua prima attestazione, registrata in un volgarizzamento trecentesco del Trattato di agricoltura di Piero de' Crescenzi, la parola viene in realtà impiegata nel significato più ristretto e specifico di 'striscia di terreno tra un filare e l'altro di viti' ("Poscia se la terra avvignata dee essere coltivata dagli uomini con marre, dee essere distanza da un tramite all'altro tre piedi o quattro", fonte: TLIO). A partire da tale significato, ancora oggi attestato in alcune zone della Toscana (come documentato dall'Atlante Lessicale Toscano), si sarebbe in seguito diffuso, per estensione, quello più generico di 'via di passaggio, sentiero'. Per quest'ultimo il GDLI riporta esempi dal primo Cinquecento alla fine dell'Ottocento, quando l'uso del termine in tale significato comincia a essere percepito come più proprio "del linguaggio scritto" (come segnala il Tommaseo-Bellini): alla fine del secolo lo si ritrova infatti quasi esclusivamente nella scrittura arcaizzante di autori come Carducci e D'Annunzio (per esempio nella raccolta Giambi ed Epodi del primo, "Qual ne l'incerto tramite / gittava il cavaliero il verde manto serico / de la sua donna al pie"; o nel romanzo Le vergini delle rocce del secondo, "Cercammo fra le macerie un tramite per giungere alla basilica diruta"), per divenire poi sempre meno frequente nel corso dei successivi decenni e infine uscire quasi del tutto dall'uso (i dizionari sincronici, tra cui lo Zingarelli, il Garzanti e il Sabatini-Coletti lo registrano infatti come raro e letterario).

Ancora vivo risulta al contrario l'uso figurato della parola nel significato di 'elemento di connessione e collegamento (riferito a cose o istituzioni); intermediario (detto di persone)', anch'esso attestato dal XIV secolo, che ricorre oggi specialmente all'interno della locuzione fare/agire da tramite: i dizionari dell'uso lo esemplificano per es. con "aprire un nuovo tramite al commercio", "un'opera che servì di tramite tra la cultura orientale e l'occidentale" (Vocabolario Treccani online e Devoto-Oli 2org), "il mare fa più da tramite che da separazione tra l'Italia e l'Albania", "Paolo è stato il tramite della nostra conoscenza" (Sabatini-Coletti 2008).

Proprio a quest'ultimo valore figurato del termine è da ricondurre la formazione della locuzione preposizionale per il tramite di nel significato di 'per mezzo di, attraverso', che si sarebbe diffusa e 
affermata soprattutto in ambito burocratico a partire dalla seconda metà dell'Ottocento: il primo a registrarne l'impiego è Giuseppe Rigutini nel suo repertorio di Neologismi buoni e cattivi piu frequenti nell'uso odierno (pubblicato nel I886), che con queste parole condanna il dilagare nella lingua del tempo del sostantivo tramite, citandone appunto l'uso anche all'interno della locuzione in questione:

Tramite è un latinismo che fu in passato rarissimo negli scrittori. [...] Ma oggi se ne fa abuso in certi linguaggi, solo per amore di singolarità o di novità, e si dice, per es. da' chirurghi: Il tramite d'una ferita ; dai pubblicisti: L'istmo di Suez è il tramite del commercio fra l'Europa e l'Oriente ; e dai burocratici: Un affare deve fare il suo tramite; Per il tramite di questo o quell'ufficio, e sim.

L'opposizione dei puristi non ne impedisce tuttavia il rapido consolidamento nell'uso (probabilmente favorito dall'analogia con la costruzione di significato equivalente per mezzo di), tanto che la locuzione viene registrata come "comune" già dai lessicografi della prima metà del secolo successivo (a partire dalla prima edizione del Novissimo dizionario della lingua italiana di Fernando Palazzi, del I939), anche se limitatamente all'ambito burocratico, in cui risulta particolarmente frequente soprattutto all'interno di formule quali per il tramite del competente ufficio, per il tramite di un ente pubblico e simili. Dall'ambito di origine, la locuzione si sarebbe in seguito in parte estesa anche alla lingua corrente (ed è infatti accolta senza restrizioni dalla maggior parte dei dizionari sincronici contemporanei), diffondendosi in particolare nella lingua giornalistica, che è in genere maggiormente soggetta all'influsso del linguaggio burocratico. Numerose sono di conseguenza le occorrenze del costrutto rilevate negli archivi dei principali quotidiani nazionali, per esempio:

E poi succede che incontro un ragazzo: cominciamo a parlarci per il tramite di una lettura, alla quale è lui ad iniziarmi, 'Adolph' di Benjamin Constant, storia di una donna che viene distrutta dall'amore per un uomo più giovane... (Anna Maria Mori, Stefania Sandrelli 'mai più patetica', "la Repubblica", I/8/1995)

È il figlio Vincenzo a parlare di Federico Del Prete, sindacalista ucciso nel 2002 per aver denunciato il pizzo riscosso dalla camorra presso gli ambulanti per il tramite di un vigile urbano. (Stella Cervasio, I superstiti, figli di una Gomorra minore, "la Repubblica", 20/10/2008)

L'investitura nobiliare l'aveva conseguita per il tramite di una serie d'artificiose telefonate, pervenute in albergo prima del suo arrivo, che chiedevano pressantemente della presenza del conte Lopez. (Tonino De Cesare, \#estatedellavita - Verso la Romagna delle notti focose (ma solo in sogno), laStampa.it, sez. Cultura, I/9/2016)

Alla più ampia locuzione preposizionale per il tramite di (regolarmente costruita nella forma preposizione + sostantivo + sintagma preposizionale, in maniera del tutto analoga ad altre locuzioni, quali per mezzo di, di fronte a, a causa di, ecc.) si sarebbe inoltre presto affiancato, come variante concorrente, l'uso del solo tramite con funzione di preposizione reggente un oggetto diretto (per esempio "tramite il competente ufficio", "invia il documento tramite fax", "gli diede sue notizie tramite un amico"): la sua origine è probabilmente da ricercare nelle esigenze di brevità proprie di alcune tipologie di scrittura, che potrebbero aver indotto alla riduzione della locuzione al solo sostantivo, forse anche per analogia con le preposizioni di significato equivalente mediante e attraverso. L'origine burocratica del costrutto è confermata dalle indicazioni dei lessicografi che per primi ne registrano l'impiego: il primo in assoluto è Alfredo Panzini, che nell'edizione del 1942 del suo Dizionario moderno (s.v. tramite) rileva, biasimandolo, l'uso del termine "nella espressione burocratica per il tramite (di codesto ufficio), o, peggio, tramite codesto ufficio", a differenza del più tardo Vocabolario di MiglioriniCappuccini, del 1965, che pur sottolineandone il carattere burocratico, si astiene da ogni valutazione di merito ("Nel ling. burocr.: Il Ministero comunicherá la risposta per il tramite (per mezzo) della Prefettura, o anche: tramite la Prefettura"). 
Anche in questo caso, analogamente a quanto avvenuto per la locuzione per il tramite di, si è assistito alla crescente diffusione del costrutto, che dall'ambito burocratico di origine si è esteso anche alla lingua corrente: il Palazzi-Folena lo registra come comune, senza restrizioni d'uso, già nel I973 ("com.fig. per il tramite, o semplicemente tramite il per mezzo, per via"), e negli stessi anni se ne rileva la presenza (attestata dal GDLI) anche nella scrittura saggistica o letteraria di autori quali Carrà, Bassani e Manganelli:

Comunicare tramite l'arte del resto fu ognora / la mia ambizione suprema. (Giorgio Bassani, Epitaffio, 1974)

Essi considerano la pittura [...] non più come un gioco meccanico di linee e di colori, ma piuttosto come una necessità dell'animo umano, cioè come un fatto che sorge dal ceppo dell'uomo tramite atti umani. (Carlo Carrà, Tutti gli scritti, 1978)

La visione è permanente, duratura, assoluta, e rivela, tramite la fame, come già tramite il gemito, il segno vocale della esistenza. (Giorgio Manganelli, Rumori o voci, 1987)

Se poi passiamo a considerare le indicazioni lessicografiche contemporanee, notiamo che, con l'eccezione del solo Vocabolario Treccani online, che lo segnala ancora come "improprio", l'uso di tramite come preposizione è accolto dalla totalità dei dizionari sincronici, segno dell'avvenuta grammaticalizzazione, documentata anche dalla registrazione nelle grammatiche più recenti: tra queste, per esempio, la Grande grammatica italiana di consultazione (Renzi-Salvi-Cardinaletti I995) considera l'uso preposizionale di tramite del tutto equivalente a quello della locuzione per tramite di, mentre quella di Trifone-Palermo del 20oI include tramite tra le preposizioni improprie legittimamente in uso in italiano, ossia quelle forme che nel loro valore primario sono avverbi (come dietro, sopra, attraverso), aggettivi (per esempio lungo), participi (mediante, durante) o appunto sostantivi, ma che possono essere impiegati anche con funzione di preposizione. L'evoluzione che ha interessato l'uso della nostra voce - che a partire dal suo valore originario di sostantivo ha iniziato a essere utilizzata prima in combinazione con altri elementi, a formare una locuzione preposizionale, e poi singolarmente, con valore di preposizione - rappresenta di conseguenza una possibilità ammessa dal nostro sistema grammaticale: non ci sono quindi ragioni per condannare il ricorso a tramite con quest'ultimo valore.

Quanto alla sua sostituibilità con le preposizioni semanticamente affini mediante e attraverso, possiamo considerare le tre forme grosso modo equivalenti, come già illustrato da Giuseppe Patota in una risposta pubblicata sul numero 48 (I, 20I4) della nostra rivista "La Crusca per voi" (p. I4): quando impiegato con valore preposizionale, tramite assume, come abbiamo visto, il significato di 'per mezzo di, attraverso' e può riferirsi sia a cosa, sia a persona, come esplicitato dal Sabatini-Coletti (che lo glossa con "per mezzo di qualcosa o di qualcuno", esemplificandone l'uso con "rispondere tramite telegramma", "tenersi in contatto tramite un amico comune").

Anche attraverso, che è nel suo valore originario un avverbio, dal significato di 'trasversalmente, obliquamente', può essere usato come preposizione, nel significato proprio di 'da una parte all'altra, in mezzo a, dentro' (per esempio "passare attraverso la siepe"), o in quello figurato di "mediante, per mezzo di', riferibile anch'esso sia a cosa, sia a persona ("il soggetto del dipinto è reso attraverso colori forti", "attraverso Giulia aveva potuto frequentare l'ambiente che lo interessava"). Analogo valore strumentale è infine espresso da mediante, che nasce come forma del participio presente di mediare, ma è oggi impiegato unicamente come preposizione, nel significato di 'per mezzo di, con l'aiuto di', di nuovo riferibile sia a cosa, sia a persona, secondo quanto possiamo ricavare dall'esemplificazione 
offerta dai dizionari dell'uso, che non danno tuttavia indicazioni esplicite in merito ("il meccanismo si mette in moto mediante una leva", "è riuscito mediante la propria buona volontà", "mediante lui ho ottenuto subito tutto").

Infine, per quanto riguarda la possibilità di declinare al plurale la forma tramite (ammessa solo quando questa è impiegata con funzione di sostantivo), la voce in questione non è registrata come invariabile da alcun dizionario dell'uso e può di conseguenza essere impiegata anche al plurale, che viene formato, analogamente ad altri sostantivi maschili in -e, con la desinenza - $i$ (e dunque il tramite > $i$ tramiti, come esplicitamente indicato dal Garzanti o dal GRADIT). È tuttavia innegabile che nel suo significato figurato di 'elemento di connessione o collegamento; intermediario', che è del resto l'unico in cui la forma tramite nel suo valore di sostantivo risulta ancora attestata nellitaliano contemporaneo, il termine venga usato prevalentemente al singolare, anche quando si riferisca a più di un elemento. Questo perché gli elementi plurali a cui si fa riferimento vengono di norma considerati nel loro valore collettivo d'insieme, di somma di elementi che svolgono la medesima funzione di collegamento e intermediazione, che viene quindi indicata da un sostantivo (tramite) che resta declinato al singolare (forse anche per influsso del suo uso come preposizione), come si può rilevare nelle seguenti attestazioni riscontrate in Google Libri:

Importante in questo contesto è stato anche il ruolo degli intellettuali, i quali affascinati dal "modello americano" sono stati il tramite per il suo radicamento fra la popolazione europea. (Pietro Neglie, Un secolo di anti-Europa. Classe, nazione e razza: la sfida totalitaria, Rubbettino Editore, 2003, p. 135)

I colloqui tutoriali sono stati il tramite per la costruzione di Unità di Apprendimento che fossero più adatte alle esigenze personali di ciascuna alunna. (Giuseppe Zanniello, Innovazione nella scuola, Armando Editore, 2006, p. I02)

Le raccolte di testi di viaggio e di pellegrinaggio in Terrasanta si pongono come base per indagini storiche sia sul complesso, sia sui personaggi che hanno fatto da tramite con le riproduzioni, in qualità di committenti, di progettisti o rilevatori di misure, di semplici devoti. (Renata Salvarani, La fortuna del Santo Sepolcro nel Medioevo: spazio, liturgia, architettura, Editoriale Jaca Book, 2008, p. 33)

Non mancano comunque attestazioni della forma plurale, seppure decisamente più sporadiche: nel PTTLIN si riscontrano per esempio 5 occorrenze di tramiti, in romanzi editi tra il 1969 e il I989, di autori quali Pietro Chiara, Carmelo Samonà, Gesualdo Bufalino e Salvatore Mannuzzu; e qualche attestazione più recente si rinviene anche in Google Libri, per esempio:

Quali sono le categorie e le forme teoriche che hanno elaborato e definito questo passaggio? E quali $i$ tramiti attraverso i quali questo passaggio si compie? Tramiti di questo passaggio, in quanto contemporaneamente interpreti e promotori della crisi della orientalizzazione della società, sono i nichilismi [...]. (Pasquale Serra, Americanismo senza America: intellettuali e identitá collettive dal 1960 ad oggi, Bari, Edizioni Dedalo, 2002, p. I20)

\section{Nota bibliografica:}

- Bruno Migliorini, Giulio Cappuccini, Vocabolario della lingua italiana, Torino, Paravia, 1965.

- Fernando Palazzi, Novissimo dizionario della lingua italiana, Milano, Ceschina, 1939.

- Fernando Palazzi, Gianfranco Folena, Novissimo dizionario della lingua italiana, Milano, Ceschina, I973. 
- Alfredo Panzini, Dizionario moderno delle parole che non si trovano nei dizionari comuni, Milano, Hoepli, I942.

- Giuseppe Rigutini, Neologismi buoni e cattivi piú frequenti nell'uso odierno, Roma, Libreria Editrice Carlo Verdesi, I886.

- Pietro Trifone, Massimo Palermo, Grammatica italiana di base, Bologna, Zanichelli, 200 .

\section{Cita come:}

Sara Giovine, Vi rispondiamo... per il giusto tramite , "Italiano digitale", 2020, XII, 2020/1 (gennaiomarzo)

DOI: $10.35948 / 2532-9006 / 2020.3227$

Copyright 2020 Accademia della Crusca

Pubblicato con licenza creative commons CC BY-NC-ND 